\title{
Seleção de Trichogramma spp., para o controle de Ecdytolopha aurantiana, com base na biologia e exigências térmicas
}

\author{
Rosa M. S. Molina ${ }^{1}$, Vanoli Fronza ${ }^{2} \&$ José R. P. Parra $^{1}$
}

${ }^{1}$ Departamento de Entomologia, Fitopatologia e Zoologia Agrícola, Escola Superior de Agricultura "Luiz de Queiroz", Universidade de São Paulo. Avenida Pádua Dias, 11, Caixa Postal 9, 13418-900 Piracicaba-SP, Brasil. rmsmolina@ig.com.br e jrpparra@esalq.usp.br

${ }^{2}$ Empresa de Pesquisa Agropecuária de Minas Gerais, Centro Tecnológico do Triângulo e Alto Paranaíba. Caixa Postal 351, $38001-970$ UberabaMG, Brasil. vanoli@epamig.br

\begin{abstract}
Biology and thermal requirements to Trichogramma spp. selection for Ecdytolopha aurantiana control. The purpose of this work was to evaluate the potential of Trichogramma atopovirilia Oatman \& Platner, 1983 and T. pretiosum Riley, 1879 as agents of control of Ecdytolopha aurantiana (Lima, 1927) (Lepidoptera: Tortricidae), an important Citrus pest in São Paulo State (South-East Brazil). In order to provide subsidies to programs of biological control with these parasitoids, studies of biology in different temperatures, thermal requirements and parasitism capacity were carried out. The temperatures $\left(18,20,22,25,28,30\right.$, and $\left.32^{\circ} \mathrm{C}\right)$ did not affect the sex ratio, however, female longevity of both species was higher at 22 and $25^{\circ} \mathrm{C}$. The temperature of $25^{\circ} \mathrm{C}$ tended to be more suitable to both emergency rate and female longevity. The egg-adult period for both Trichogramma species was inversely proportional to temperature. The thermal requirements of the two species were very close, about 108 DD (degree days). Neither the natural rearing host, E. aurantiana, nor the alternative host Anagasta kuehniella (Zeller, 1879) (Lepidoptera, Pyralidae), affected the number of parasitized eggs per Trichogramma female. The parasitism rate and the number of emerged adults per egg on E. aurantiana eggs were higher than on A. kuehniella eggs. However, the emergency rate was higher when the parasitoids were reared on A. kuehniella eggs. Both Trichogramma species could be tested in the field for citrus fruit borer control. The thermal requirements and the parasitism capacity also could be good parameters for selection of Trichogramma species/strains.
\end{abstract}

KEYwords. Biological control; citrus fruit borer; parasitism; Trichogramma atopovirilia; Trichogramma pretiosum.

Resumo. Seleção de Trichogramma spp., para o controle de Ecdytolopha aurantiana, com base na biologia e exigências térmicas. O objetivo deste trabalho foi avaliar o potencial de utilização de Trichogramma atopovirilia Oatman \& Platner, 1983 e T. pretiosum Riley, 1879 como agentes de controle de Ecdytolopha aurantiana (Lima, 1927) (Lepidoptera: Tortricidae), importante praga da citricultura paulista. Foram realizados estudos de biologia em diferentes temperaturas, exigências térmicas e capacidade de parasitismo, visando fornecer subsídios a programas de controle biológico com estes parasitóides. A razão sexual não foi afetada pelas temperaturas estudadas $\left(18,20,22,25,28,30\right.$ e $\left.32^{\circ} \mathrm{C}\right)$, porém, a longevidade de fêmeas das duas espécies foi maior a 22 e $25^{\circ} \mathrm{C}$. Para a porcentagem de emergência e longevidade de fêmeas, a temperatura que tendeu a ser mais adequada foi a de $25^{\circ} \mathrm{C}$. A duração do período ovo-adulto para as duas espécies foi inversamente relacionada com o aumento da temperatura, sendo as exigências térmicas dessas espécies muito próximas, em torno de 108 GD. O hospedeiro natural, E. aurantiana, ou o alternativo, Anagasta kuehniella (Zeller, 1879) (Lepidoptera, Pyralidae), não afetaram o número de ovos parasitados por fêmea de Trichogramma. Porém, a porcentagem de parasitismo e o número de adultos emergidos por ovo, quando provenientes de ovos de E. aurantiana, foram maiores do que os criados sobre ovos de A. kuehniella. A porcentagem de emergência foi, no entanto, maior quando os parasitóides foram provenientes de ovos de A. kuehniella. As duas espécies de Trichogramma estudadas poderão ser testadas em campo para controle do bicho-furão-dos-citros, havendo uma indicação de que as exigências térmicas também podem servir como um bom parâmetro para seleção de espécies e/ou linhagens de Trichogramma, aliadas ao estudo de capacidade de parasitismo.

Palavras-Chave. Bicho-furão-dos-citros; controle biológico; parasitismo; Trichogramma atopovirilia; Trichogramma pretiosum.

As pragas estão entre os fatores limitantes para a produção de citros no Brasil, exigindo muita mão-de-obra para seu monitoramento e um alto volume de agroquímicos para seu controle. Dentre tais pragas, nos últimos anos, especialmente em São Paulo, vem se destacando o bicho-furão-dos-citros, Ecdytolopha aurantiana (Lima, 1927) (Lepidoptera, Tortricidae). Este inseto provoca perda total do fruto atacado, provocando sua queda e apodrecimento, não permitindo seu aproveitamento nem para a indústria e nem para o consumo 'in natura'. Estimaram-se que as perdas devido ao ataque desta praga sejam de até 50 milhões de dólares por ano (Fundecitrus 2000).

Os estudos básicos de biologia (Garcia \& Parra 1999) e comportamento (Bento et al. 2001) resultaram na síntese do feromônio sexual do bicho-furão, essencial para o monitoramento populacional da praga. Segundo Bento et al. (2001), a utilização deste feromônio pelos agricultores reduziu sensivelmente o número de aplicações de inseticidas na cultura de citros, pois muitas aplicações eram feitas de forma preventiva ou em épocas inadequadas. 
O uso de inimigos naturais como componente adicional em programas de manejo do bicho-furão também pode ser viável, pois pesquisas no Brasil demonstraram o potencial de controle de E. aurantiana com Trichogramma pretiosum Riley, 1879 (Hymenoptera, Trichogrammatidae) (Parra \& Zucchi 2004).

Desta forma, o objetivo do presente trabalho foi avaliar a possibilidade de seleção de linhagens de Trichogramma spp., como agente de controle de E. aurantiana, com base em avaliações biológicas, incluindo biologia em diferentes temperaturas, exigências térmicas e parasitismo, visando a sua liberação para controle desta importante praga da citricultura paulista e atuando como mais um componente do seu manejo.

\section{MATERIAL E MÉTODOS}

Criação dos parasitóides e hospedeiros. As fêmeas de Trichogramma atopovirilia Oatman \& Platner, 1983 e $T$. pretiosum Riley, 1879 e os ovos de Ecdytolopha aurantiana (Lima, 1927) e Anagasta kuehniella (Zeller, 1879) (Lepidoptera, Pyralidae) utilizados nos experimentos foram obtidos das criações mantidas no Laboratório de Biologia de Insetos do Departamento de Entomologia, Fitopatologia e Zoologia Agrícola, da Escola Superior de Agricultura "Luiz de Queiroz", Universidade de São Paulo (ESALQ/USP). As criações foram conduzidas segundo as metodologias de Parra (1997), para a traça e os parasitóides, e de Garcia \& Parra (1999) para E. aurantiana.

Biologia em diferentes temperaturas. Antes da instalação do experimento, as duas espécies de Trichogramma foram mantidas, por uma geração, em ovos de E. aurantiana, a fim de eliminar um provável condicionamento pré-imaginal pela criação no hospedeiro alternativo, A. kuehniella. Fêmeas de cada espécie, com 24 horas de idade, foram individualizadas em tubos de vidro $(12 \times 75 \mathrm{~mm})$. Ofereceram-se, para cada fêmea, 30 ovos de E. aurantiana com, no máximo, 24 horas de idade. Como fonte de alimento, utilizou-se uma gotícula de mel puro. O parasitismo foi permitido por 24 horas em câmaras climatizadas, reguladas a $25 \pm 1^{\circ} \mathrm{C}$, UR: $70 \pm 10 \%$ e fotofase de 14 horas. Após este período, os parasitóides foram retirados dos tubos e nestes foi colocado algodão umedecido com sulfato de cobre a $1 \%$ para evitar ressecamento e crescimento de fungos nos ovos, sendo em seguida tampados com algodão hidrófilo. Os tubos foram então transferidos para câmaras climatizadas, reguladas com temperaturas selecionadas (18, 20, 22, 25, 28, 30 e $32^{\circ} \mathrm{C}$ ), com UR: $70 \pm 10 \%$ e fotofase de 14 horas. Para cada uma das sete temperaturas foram utilizadas cinco repetições de cada espécie estudada, sendo que cada repetição foi constituída por uma parcela com cinco tubos. Foram avaliados os seguintes parâmetros: duração do desenvolvimento (ovo-adulto), porcentagem de emergência, número de adultos emergidos por ovo, longevidade de fêmeas e razão sexual.

Determinação das exigências térmicas. $O$ limiar térmico inferior de desenvolvimento, ou temperatura base $(\mathrm{Tb})$, e a constante térmica $(\mathrm{K})$, foram calculados pelo método da hipérbole, com base na duração do período ovo-adulto, obtido nas diferentes temperaturas, para as duas espécies de Trichogramma, utilizando-se o programa MOBAE (Modelos Estatísticos Aplicados à Entomologia) (Haddad et al. 1999).

Capacidade de parasitismo. Avaliou-se a capacidade de parasitismo, sobre ovos de bicho-furão, de fêmeas de $T$. atopovirilia e $T$. pretiosum provenientes de ovos de $E$. aurantiana e de A. kuehniella, em câmara climatizada regulada a $28 \pm 1^{\circ} \mathrm{C}$, UR: $70 \pm 10 \%$ e fotofase de 14 horas.

Foram individualizadas fêmeas de cada espécie de Trichogramma, provenientes de cada hospedeiro, alimentadas com mel puro em tubos de vidro $(12 \times 75 \mathrm{~mm})$ tampados com filme plástico de PVC. Cada espécie foi representada por cinco repetições, sendo cada repetição constituída por uma parcela com cinco tubos. Trinta ovos foram oferecidos diariamente, a cada fêmea e, após o período de 24 horas de parasitismo, os ovos foram transferidos para tubos de vidro contendo um chumaço de algodão umedecido com sulfato de cobre a $1 \%$, sendo em seguida tampados com algodão hidrófilo. $\mathrm{O}$ parasitismo foi avaliado até a morte das fêmeas. Foram avaliados e calculados o número de ovos parasitados diariamente, a porcentagem acumulada de parasitismo, o número total de ovos parasitados por fêmea, a longevidade das fêmeas, a porcentagem de emergência e o número de adultos emergidos por ovo.

Os parâmetros biológicos avaliados foram submetidos à análise de variância e as médias comparadas pelo teste de Tukey, ao nível de 5\% de probabilidade, utilizando-se o programa SAS (SAS Institute 1999). Os dados de porcentagem de emergência foram transformados em arc sen $\sqrt{(\mathrm{x} / 100)}$ e a longevidade de fêmeas em $\sqrt{(\mathrm{x}+1)}$.

\section{RESULTADOS E DISCUSSÃO}

Biologia. Houve efeito significativo da interação espécie $\mathrm{x}$ temperatura para a porcentagem de emergência, duração do período ovo-adulto (Tabela I) e longevidade de fêmeas (Tabela II), indicando resposta diferenciada nestas características, entre as espécies, quando submetidas às diferentes temperaturas.

Foi observada redução significativa na porcentagem de emergência de Trichogramma pretiosum Riley, 1879, em relação à T. atopovirilia Oatman \& Platner, 1983, nas temperaturas de 18,30 e $32^{\circ} \mathrm{C}$ (Tabela I). Para T. atopovirilia observou-se maior porcentagem de emergência na faixa entre 22 a $32^{\circ} \mathrm{C}$, variando de 47,3 a $58,9 \%$. Para $T$. pretiosum, os maiores valores de porcentagem de emergência foram observados nas temperaturas de 22 e $25^{\circ} \mathrm{C}$, respectivamente, 50,8 e $55,9 \%$, as quais diferiram significativamente apenas dos valores observados a 18 e $32^{\circ} \mathrm{C}$.

A duração média do período ovo-adulto de T. atopovirilia e de T. pretiosum sobre ovos de E. aurantiana foi afetada pela temperatura. Como era de se esperar, nas condições estudadas ocorreu um aumento significativo da velocidade 

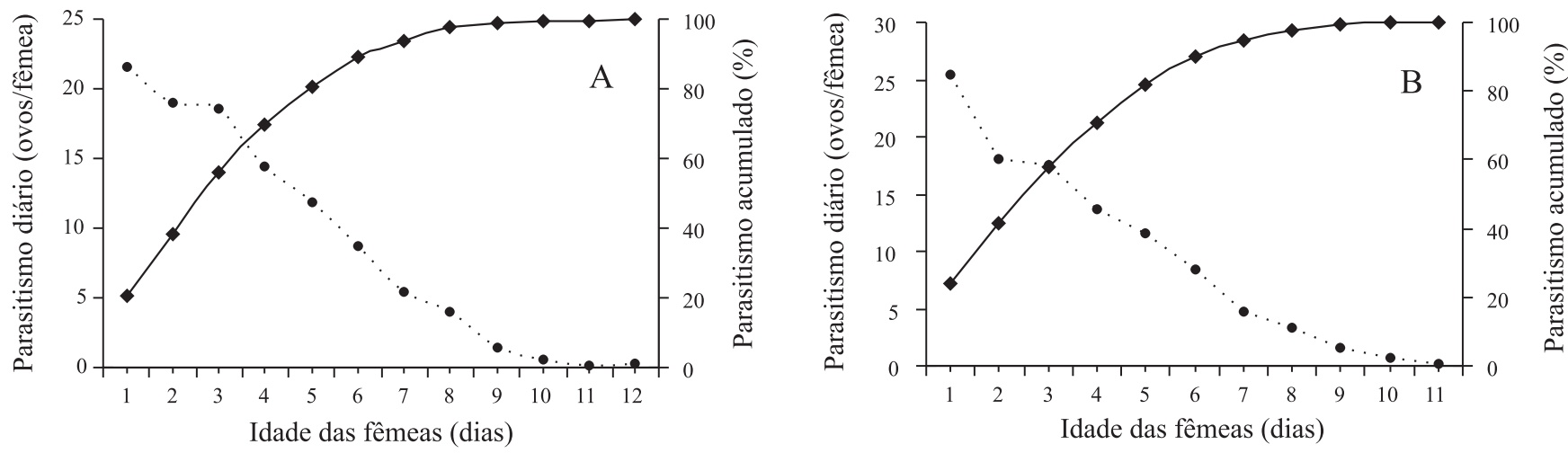

Fig. 1. Parasitismo diário (-) e parasitismo acumulado (-) de duas espécies de Trichogramma provenientes de ovos de Ecdytolopha aurantiana. Temperatura: $28 \pm 1^{\circ} \mathrm{C}$, UR: $70 \pm 10 \%$ e fotofase $14 \mathrm{~h}$. A. T. atopovirilia; B. T. pretiosum.

de desenvolvimento com a elevação térmica (Tabela I). Vários autores também constataram a influência da temperatura sobre a velocidade de desenvolvimento deste parasitóide (ButlerJunior \& Lopez 1980; Yu et al. 1984; Bleicher \& Parra 1990a; Pratissoli \& Parra 2000).

Embora, numericamente, tenha ocorrido uma tendência de aumento da duração do período ovo-adulto de T. atopovirilia em relação à $T$. pretiosum, houve diferenças estatísticas entre as duas espécies apenas nas temperaturas de 18 e $20^{\circ} \mathrm{C}$ (Tabela I). Os valores obtidos nesta pesquisa para $T$. pretiosum assemelham-se àqueles apresentados por Bleicher \& Parra (1989), na mesma faixa de temperatura.

Aparentemente, espécies diferentes, ou de locais diferentes, têm longevidades diferentes, em função da variação térmica e da alimentação (Bleicher \& Parra 1989). Esta reação diferenciada ficou evidenciada nas espécies estudadas, pois a longevidade das fêmeas de $T$. atopovirilia foi maior nas temperaturas de 20,22 e $25^{\circ} \mathrm{C}$, com valores de $8,7,12,6$ e 10 dias, respectivamente (Tabela II). T. pretiosum apresentou maior longevidade para fêmeas a 22 e $25^{\circ} \mathrm{C}$, com 9,5 e 9,1 dias, respectivamente, havendo diferenças entre espécies nas temperaturas de $20,22,30$ e $32^{\circ} \mathrm{C}$ para longevidade de fêmeas.
Segundo Bleicher \& Parra (1989) observou-se maior longevidade na faixa de 20 a $22^{\circ} \mathrm{C}$, devido à redução na atividade metabólica do inseto. Segundo estes autores, a diminuição destes processos abaixo de certo limite teria efeitos negativos, o que explicaria a diminuição na longevidade a $18^{\circ} \mathrm{C}$ observada para $T$. pretiosum e também no presente trabalho para a longevidade de fêmeas das duas espécies estudadas (Tabela II). Russo \& Voegelé (1982) observaram, em estudos feitos entre $11 \mathrm{e} 34^{\circ} \mathrm{C}$, que baixas e altas temperaturas tiveram efeito negativo na longevidade de algumas espécies de Trichogramma.

Com relação à razão sexual e ao número de adultos emergidos por ovo nas espécies estudadas, não houve efeito significativo da interação espécie x temperatura e nem do efeito geral (na média das duas espécies) da temperatura, observandose apenas diferença significativa entre as duas espécies (na média das sete temperaturas) para a razão sexual (Tabela II). Outros pesquisadores (Calvin et al. 1984; Bleicher \& Parra 1989) também não observaram efeito da temperatura sobre a razão sexual de populações de Trichogramma em diferentes hospedeiros.
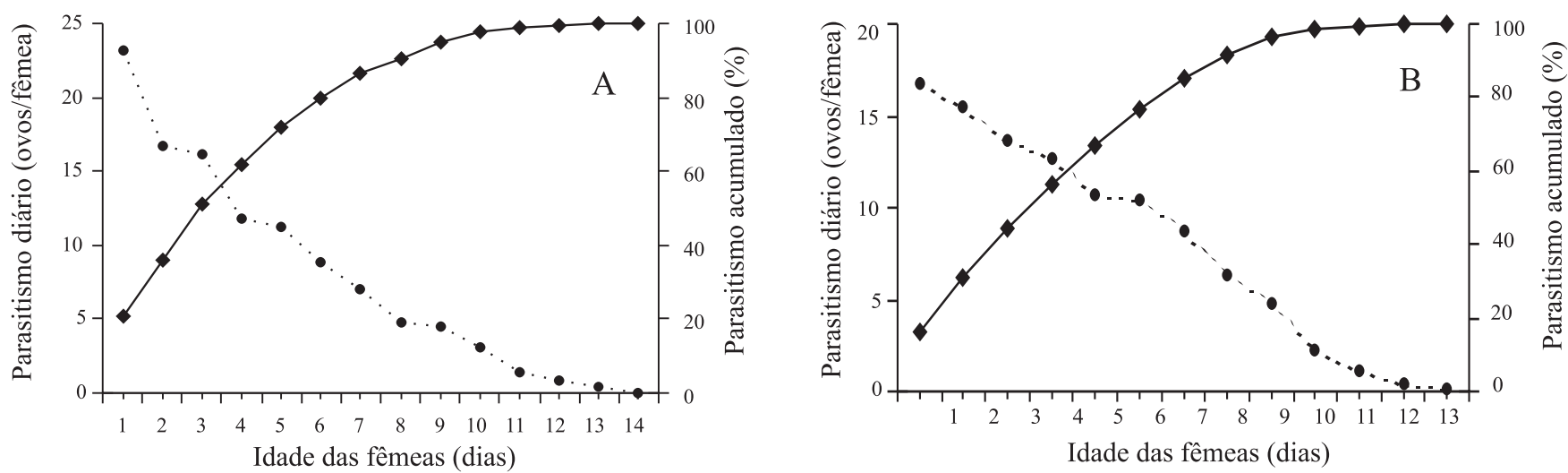

Fig. 2. Parasitismo diário (-.) e parasitismo acumulado (-) de duas espécies de Trichogramma provenientes de ovos de Anagasta kuehniella. Temperatura: $28 \pm 1{ }^{\circ} \mathrm{C}$, UR: $70 \pm 10 \%$ e fotofase $14 \mathrm{~h}$. A. T. atopovirilia; B. T. pretiosum. 
Tabela I. Emergência (\%) e duração do ciclo (ovo-adulto) de Trichogramma atopovirilia e T. pretiosum, em diferentes temperaturas, criados em ovos de Ecdytolopha aurantiana. Parasitismo: $24 \mathrm{~h}$, UR: $70 \pm 10 \%$ e fotofase $14 \mathrm{~h}$.

\begin{tabular}{|c|c|c|c|c|}
\hline \multirow{2}{*}{$\begin{array}{l}\text { Temp. } \\
\left({ }^{\circ} \mathrm{C}\right)\end{array}$} & \multicolumn{2}{|c|}{ Emergência (\%) } & \multicolumn{2}{|c|}{ Duração (ovo-adulto) } \\
\hline & T. atopovirilia & T. pretiosum & T. atopovirilia & T. pretiosum \\
\hline 18 & $42,5 \pm 8,6$ b A & $30,5 \pm 8,6 \quad$ b $\quad B$ & $23,6 \pm 1,6 \mathrm{a} \quad \mathrm{A}$ & $21,0 \pm 2,0 \mathrm{a} \quad \mathrm{B}$ \\
\hline 20 & $40,9 \pm 5,3$ b A & $41,9 \pm 5,3 \mathrm{ab} \quad \mathrm{A}$ & $16,1 \pm 1,6 \mathrm{~b} \quad \mathrm{~A}$ & $14,9 \pm 0,8 \quad \mathrm{~b} \quad \mathrm{~B}$ \\
\hline 22 & $47,3 \pm 8,5 \mathrm{ab} A$ & $50,8 \pm 8,5$ a $\quad A$ & $15,4 \pm 0,4 \mathrm{~b} \quad \mathrm{~A}$ & $14,6 \pm 0,3 \quad \mathrm{~b} \quad \mathrm{~A}$ \\
\hline 25 & $54,3 \pm 9,9 \mathrm{ab} \mathrm{A}$ & $55,9 \pm 9,9$ a $\quad \mathrm{A}$ & $8,7 \pm 1,4$ c $\mathrm{A}$ & $7,9 \pm 0,7 \quad$ c $\mathrm{A}$ \\
\hline 28 & $51,0 \pm 5,6 \mathrm{ab} A$ & $43,2 \pm 5,6 \mathrm{ab} \quad \mathrm{A}$ & $7,1 \pm 0,4$ c $\mathrm{A}$ & $7,0 \pm 0,1$ \\
\hline 30 & $58,9 \pm 6,0$ a $\mathrm{A}$ & $43,0 \pm 6,0 \mathrm{ab} \quad \mathrm{B}$ & $6,3 \pm 0,2 \quad \mathrm{dA}$ & $6,1 \pm 0,1$ \\
\hline 32 & $53,5 \pm 5,6 \mathrm{ab} A$ & $39,9 \pm 5,6 \quad$ b $\quad B$ & $6,2 \pm 0,2$ & $6,0 \pm 0,2$ \\
\hline Média & 49,8 & 43,6 & 11,9 & 11,1 \\
\hline
\end{tabular}

Médias seguidas de mesma letra minúscula nas colunas e maiúscula nas linhas não diferem entre si, pelo teste de Tukey, a 5\% de probabilidade.

Em resumo, a temperatura de $25^{\circ} \mathrm{C}$ tendeu a ser mais adequada no estudo de biologia para as espécies $T$. atopovirilia e T. pretiosum para as variáveis biológicas emergência e longevidade de fêmeas.

Exigências térmicas. Com base na velocidade de desenvolvimento nas diferentes temperaturas $(18,20,22,25$, 28,30 e $32^{\circ} \mathrm{C}$ ), para o período de ovo-adulto dos parasitóides, pôde-se determinar o limite térmico inferior de desenvolvimento (Tb) e a constante térmica $(\mathrm{K})$, das espécies estudadas, em ovos de E. aurantiana (Tabela III). Assim, foram constatados os respectivos valores de $13,5^{\circ} \mathrm{C}$ e $107,5 \mathrm{GD}$ (graus dias) para T. atopovirilia e $12,9^{\circ} \mathrm{C}$ e 107,8 GD para T. pretiosum, com altos coeficientes de determinação (96,7 e 95,9\%, respectivamente).

Comparando-se os valores de $\mathrm{Tb}$ e $\mathrm{K}$ obtidos na presente pesquisa (Tabela III) com os apresentados por outros autores para diferentes linhagens/espécies de Trichogramma, constatou-se que as temperaturas bases obtidas para as duas espécies de Trichogramma foram ligeiramente superiores aos resultados apresentados por Yu et al. $(1984)\left(10^{\circ} \mathrm{C}\right)$, e semelhantes aos encontrados por Bleicher \& Parra (1990a) $\left(12,8^{\circ} \mathrm{C}\right)$ e Pratissoli \& Parra $(2000)\left(13,5^{\circ} \mathrm{C}\right)$.
As variações entre valores de temperatura base e constante térmica observadas na presente pesquisa e aquelas registradas por outros autores (Butler-Junior \& Lopez 1980; Yu et al. 1984; Bleicher \& Parra 1989; Pratissoli \& Parra, 2000), indicam a influência da espécie/linhagem e do hospedeiro sobre a biologia desses parasitóides.

Capacidade de Parasitismo. Para as características biológicas avaliadas houve significância da interação espécie $\mathrm{x}$ hospedeiro apenas para a porcentagem de emergência (Tabela IV).

A capacidade de parasitismo a $28^{\circ} \mathrm{C}$, de T. atopovirilia e $T$. pretiosum, provenientes de E. aurantiana, foi de 105,8 e 105,2 ovos/fêmea e, quando criadas em A. kuehniella, de 109,8 e 103,6, respectivamente (Tabela IV). Estes resultados estão dentro da faixa relatada por Parra \& Zucchi (1986), ou seja, de 70 a 120 ovos parasitados por fêmea para espécies de Trichogramma. Entretanto, Beserra (2004) relatou que $T$. atopovirilia apresentou maior capacidade de parasitismo do que T. pretiosum em ovos de Spodoptera frugiperda (J.E. Smith, 1797) (Lepidoptera: Noctuidae) em relação a $A$. kuehniella. Como o número de ovos parasitados por fêmea não diferiu significativamente entre as espécies e nem quanto

Tabela II. Longevidade de fêmeas, razão sexual e número de adultos emergidos por ovo de Trichogramma atopovirilia e T. pretiosum, em diferentes temperaturas, criados em ovos de Ecdytolopha aurantiana. Parasitismo: 24h, UR: $70 \pm 10 \%$ e fotofase $14 \mathrm{~h}$.

\begin{tabular}{|c|c|c|c|c|c|c|}
\hline \multirow{2}{*}{$\begin{array}{l}\text { Temp. } \\
\left({ }^{\circ} \mathrm{C}\right)\end{array}$} & \multicolumn{2}{|c|}{ Longevidade (fêmeas) } & \multicolumn{2}{|c|}{ Razão sexual } & \multicolumn{2}{|c|}{$\mathrm{N}^{o}$ de adulto/ovo } \\
\hline & T. atopovirilia & T. pretiosum & T. atopovirilia & T. pretiosum & T. atopovirilia & T. pretiosum \\
\hline 18 & $5,3 \pm 1,4 \mathrm{c} \quad \mathrm{A}$ & $5,9 \pm 1,0 \mathrm{~b} \quad \mathrm{~A}$ & $0,8 \pm 0,1$ & $0,6 \pm 0,2$ & $1,5 \pm 0,2$ & $1,7 \pm 0,3$ \\
\hline 20 & $8,7 \pm 1,3$ b A & $6,4 \pm 1,4$ b $\quad$ B & $0,6 \pm 0,1$ & $0,5 \pm 0,2$ & $1,4 \pm 0,2$ & $1,4 \pm 0,2$ \\
\hline 22 & $12,6 \pm 1,3$ a $A$ & $9,5 \pm 1,4$ a $\quad B$ & $0,7 \pm 0,1$ & $0,5 \pm 0,2$ & $1,4 \pm 0,1$ & $1,6 \pm 0,3$ \\
\hline 25 & $10,0 \pm 1,3 \mathrm{ab} A$ & $9,1 \pm 1,2$ a $\quad A$ & $0,7 \pm 0,1$ & $0,6 \pm 0,2$ & $1,6 \pm 0,2$ & $1,7 \pm 0,1$ \\
\hline 28 & $5,6 \pm 1,0$ с A & $6,2 \pm 0,9 \quad \mathrm{c} \mathrm{A}$ & $0,7 \pm 0,2$ & $0,8 \pm 0,1$ & $1,5 \pm 0,3$ & $1,5 \pm 0,1$ \\
\hline 30 & $4,2 \pm 1,2$ с B & $5,8 \pm 0,9$ b A & $0,7 \pm 0,1$ & $0,6 \pm 0,1$ & $1,5 \pm 0,1$ & $1,7 \pm 0,3$ \\
\hline 32 & $5,6 \pm 1,2 \quad$ с A & $4,2 \pm 0,4 \quad b \quad B$ & $0,7 \pm 0,1$ & $0,7 \pm 0,1$ & $1,5 \pm 0,1$ & $1,5 \pm 0,2$ \\
\hline Média & 7,4 & 6,7 & $0,7 \mathrm{~A}$ & $0,6 \mathrm{~B}$ & $1,5 \mathrm{~A}$ & $1,6 \mathrm{~A}$ \\
\hline
\end{tabular}

Médias seguidas de mesma letra minúscula nas colunas e maiúscula nas linhas não diferem entre si, pelo teste de Tukey, a 5\% de probabilidade. 

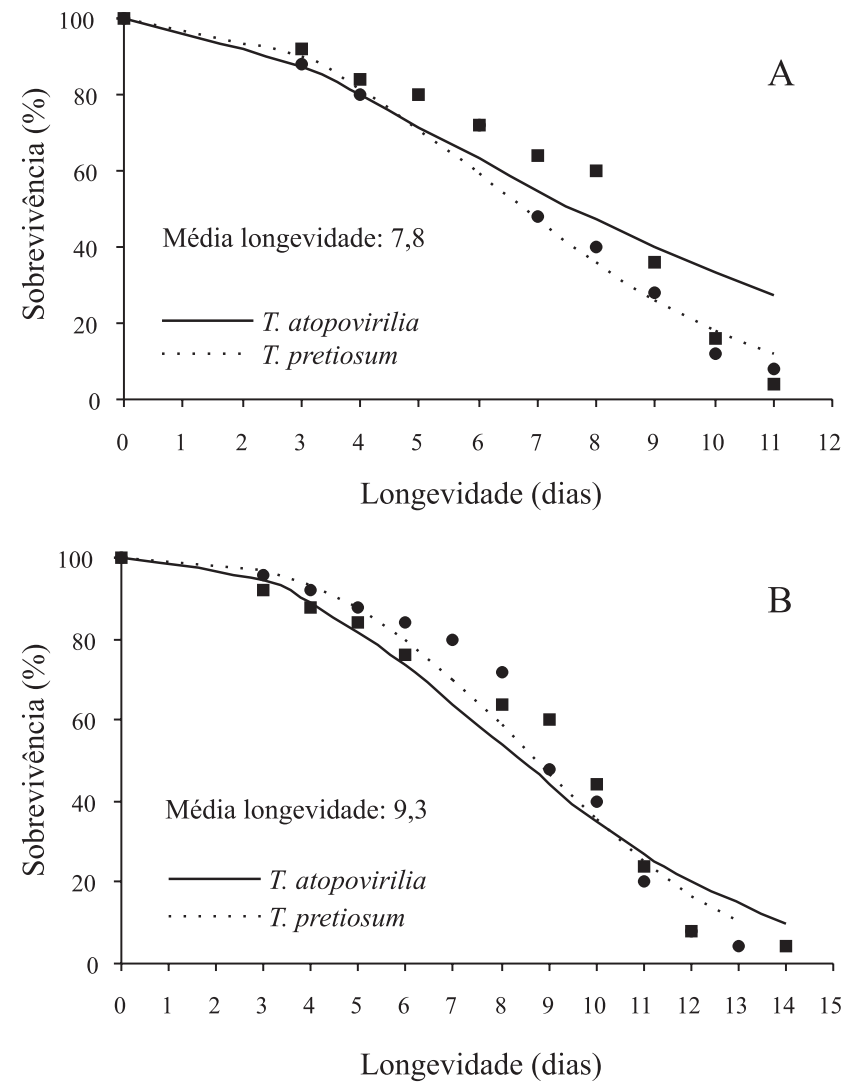

Fig. 3. Sobrevivência de adultos de Trichogramma atopovirilia e $T$. pretiosum. A. Provenientes de ovos de Ecdytolopha aurantiana. B. Provenientes de ovos de Anagasta kuehniella. Valores observados e estimados pela equação de Weilbull. Temperatura: $28 \pm 1^{\circ} \mathrm{C}$, UR: $70 \pm$ $10 \%$ e fotofase $14 \mathrm{~h}$

ao hospedeiro, também não havendo interação significativa entre estes (Tabela IV), isto indica que o hospedeiro alternativo pode ser usado para criação de T. atopovirilia e T. pretiosum.

Na literatura é relatado que a capacidade total de parasitismo é afetada pelo hospedeiro de criação, linhagem/espécie, longevidade, temperatura, suprimento alimentar, tamanho do recipiente onde se deu o parasitismo e local de coleta dos parasitóides (Parra \& Zucchi 1986; Bigler et al. 1987; Hassan \& Guo 1991; Wührer \& Hassan 1993). Como na presente pesquisa a capacidade de parasitismo foi avaliada apenas a $28^{\circ} \mathrm{C}$, não foi possível concluir se a temperatura interferiu na fecundidade do parasitóide, embora seja relatada a forte influência deste fator sobre a fecundidade de Trichogramma (Bleicher \& Parra 1989).
A porcentagem média de parasitismo total não diferiu entre espécies, mas diferiu quanto ao hospedeiro das mesmas, sendo observado 51,9\% para fêmeas de Trichogramma provenientes de ovos de E. aurantiana e 44,1\% para as provenientes de ovos de A. kuehniella (Tabela IV). Isto demonstra que o comportamento de aceitação do hospedeiro ou preferência pode ser atribuído à experiência que a fêmea adquire durante $\mathrm{o}$ desenvolvimento larval (condicionamento pré-imaginal) conforme comentado por Cobert (1985) e Kaiser et al.(1989); ou pelo aprendizado associativo ou $\alpha$-condicionamento, pelo qual as fêmeas associam estímulos novos (adquiridos) aos inatos, podendo adaptar o seu comportamento ao ambiente no qual elas conviveram quando adultas ou jovens (Kaiser et al. 1989; Vinson 1998; Nurindah et al. 1999). Porém, provavelmente, uma porcentagem de parasitismo $15 \%$ menor, encontrada no presente trabalho, não inviabilizaria o uso do hospedeiro alternativo na prática.

Quanto à emergência, houve diferença estatística entre espécies somente quando provenientes de ovos de $A$. kuehniella, com $60 \%$ para $T$. atopovirilia e $49,6 \%$ para $T$. pretiosum (Tabela IV). Comparando-se cada espécie com relação às origens, ambas as espécies de Trichogramma apresentaram emergência significativamente superior quando provenientes de ovos de A. kuehniella.

$\mathrm{O}$ número de adultos emergidos por ovo diferiu apenas quanto ao hospedeiro dos parasitóides, sendo de 1,32 quando provenientes de ovos de E. aurantiana e 1,20 de A. kuehniella (Tabela IV). Segundo Vinson (1997) há um número ideal de ovos para determinado tamanho de hospedeiro, sendo que o tamanho do hospedeiro não influencia apenas o número de ovos depositados, mas também o tamanho do adulto de Trichogramma, o qual depende dos recursos disponíveis para o desenvolvimento da larva; já a fecundidade é proporcional ao tamanho da fêmea.

Com relação à porcentagem acumulada de parasitismo, verificou-se que as fêmeas das duas espécies de Trichogramma, provenientes de ovos de E. aurantiana, foram as que parasitaram maior número de ovos em menor espaço de tempo (Figs. 1 e 2). Neste caso, aos cinco dias já tinham sido parasitados mais de $80 \%$ do total de ovos (Fig. 1); para as fêmeas provenientes de A. kuehniella este valor só foi ultrapassado por volta do sétimo dia (Fig. 2). Bleicher \& Parra (1990b) observaram ritmo de parasitismo distinto para diferentes populações de T. pretiosum. Entretanto, Sá \& Parra (1994) e Cônsoli \& Parra (1996) obtiveram um ritmo de parasitismo semelhante para linhagens de T. pretiosum, independente do hospedeiro no qual foram criados.

Tabela III. Limite térmico inferior de desenvolvimento $(\mathrm{Tb})$, constante térmica $(\mathrm{K})$ e coeficiente de determinação $\left(\mathrm{R}^{2}\right)$ do período ovo-adulto de duas espécies de Trichogramma, criadas em ovos de Ecdytolopha aurantiana, em condições de laboratório. UR: $70 \pm 10 \%$ e fotofase $14 \mathrm{~h}$.

\begin{tabular}{ccccc}
\hline Espécies & $\mathrm{Tb}\left({ }^{\circ} \mathrm{C}\right)$ & $\mathrm{K}(\mathrm{GD})$ & Equação de regressão & $\mathrm{R}^{2}(\%)$ \\
\hline T. atopovirilia & 13,5 & 107,5 & $\mathrm{Y}=-0,125569+0,009297 \mathrm{x}$ & 96,7 \\
T. pretiosum & 12,9 & 107,8 & $\mathrm{Y}=-0,120112+0,009280 \mathrm{x}$ & 95,9 \\
\hline
\end{tabular}

Médias seguidas de mesma letra minúscula nas colunas e maiúscula nas linhas não diferem entre si, pelo teste de Tukey, a 5\% de probabilidade. 
Tabela IV. Valores médios de números de ovos parasitados por fêmea, parasitismo, emergência e número de adultos emergidos por ovo, de Trichogramma atopovirilia e Trichogramma pretiosum provenientes de ovos de Ecdytolopha aurantiana e Anagasta kuehniella. Temperatura: $28 \pm 1^{\circ} \mathrm{C}$, UR: $70 \pm 10 \%$ e fotofase $14 \mathrm{~h}$.

\begin{tabular}{|c|c|c|c|c|}
\hline \multirow{2}{*}{ Características } & \multirow{2}{*}{ Espécies } & \multicolumn{2}{|c|}{ Origem } & \multirow{2}{*}{ Média } \\
\hline & & E. aurantiana & A. kuehniella & \\
\hline $\mathrm{N}^{\circ}$ de ovos & T. atopovirilia & $105,8 \pm 40,75$ & $109,8 \pm 38,24$ & $107,8 \mathrm{a}$ \\
\hline \multirow{2}{*}{$\begin{array}{l}\text { parasitados/ } \\
\text { fềmea }\end{array}$} & T. pretiosum & $105,2 \pm 47,00$ & $103,6 \pm 36,37$ & $104,4 \mathrm{a}$ \\
\hline & Média & $105,5 \mathrm{~A}$ & $106,7 \mathrm{~A}$ & \\
\hline \multirow{3}{*}{ Parasitismo (\%) } & T. atopovirilia & $51,0 \pm 16,62$ & $47,2 \pm 9,70$ & $49,1 \mathrm{a}$ \\
\hline & T. pretiosum & $52,9 \pm 10,75$ & $41,0 \pm 6,08$ & 46,9 a \\
\hline & Média & $51,9 \mathrm{~A}$ & $44,1 \mathrm{~B}$ & \\
\hline \multirow{3}{*}{ Emergência (\%) } & T. atopovirilia & $42,2 \pm 11,09$ a B & $60,0 \pm 6,84$ a A & 51,1 \\
\hline & T. pretiosum & $42,8 \pm 17,24$ a B & $49,6 \pm 5,92 \mathrm{~b} \mathrm{~A}$ & 46,2 \\
\hline & Média & 42,5 & 54,8 & \\
\hline \multirow{3}{*}{$\begin{array}{l}\mathrm{N}^{\circ} \text { de adultos } \\
\text { por ovo }\end{array}$} & T. atopovirilia & $1,29 \pm 0,33$ & $1,21 \pm 0,12$ & $1,25 \mathrm{a}$ \\
\hline & T. pretiosum & $1,35 \pm 0,27$ & $1,18 \pm 1,18$ & $1,27 \mathrm{a}$ \\
\hline & Média & $1,32 \mathrm{~A}$ & $1,20 \mathrm{~B}$ & \\
\hline
\end{tabular}

Médias seguidas de mesma letra minúscula nas colunas e maiúscula nas linhas não diferem entre si, pelo teste de Tukey, a 5\% de probabilidade.

Embora tenha sido verificada uma maior longevidade média para fêmeas provenientes de A. kuehniella ( 9,3 dias) do que de E. aurantiana (7,8 dias) (Fig. 3), o número total de ovos parasitados pelas fêmeas não diferiu estatisticamente entre os hospedeiros e nem entre as espécies (Tabela IV). No geral, houve uma compensação entre os diferentes parâmetros biológicos avaliados tanto para hospedeiros como para as espécies de Trichogramma.

Assim, as duas espécies de Trichogramma estudadas poderão ser testadas em campo para controle do bicho-furãodos-citros, ficando demonstrado que além da capacidade de parasitismo, também as exigências térmicas podem servir como um bom parâmetro para seleção de espécies e/ou linhagens de Trichogramma. Portanto, além da utilização das exigências térmicas em programas de produção massal de parasitóides (liberações inundativas) e para estimar o número de gerações de parasitóides para programas de liberações em áreas ecologicamente distintas, a determinação das exigências térmicas poderá também auxiliar na escolha de linhagens/ espécies de Trichogramma para controle de pragas.

Agradecimentos. Ao Fundo de Defesa da Citricultura FUNDECITRUS, pelo suporte financeiro da pesquisa, à $\mathrm{Dr}^{\mathrm{a}}$. Ranyse B. Querino, pela identificação das espécies de Trichogramma, à Engenheira Agrônoma, MSc. Sandra R. Magro, pelas sugestões no texto e ao Engenheiro Agrônomo, MSc. Dori E. Nava pelo apoio durante as correções do texto.

\section{REFERÊNCIAS}

Bento, J. M. S.; E. V. Vilela; J. R. P. Parra \& W. Leal. S. 2001. Monitoramento do bicho-furão-dos-citros com feromônio sexual: bases comportamentais para utilização dessa nova estratégia. Laranja 22: 351-366.

Beserra, E. B. 2004. Biologia parasitismo de Trichogramma atopovirilia Oatman \& Platner e Trichogramma pretiosum Riley (Hymenoptera, Trichogrammatidae) em ovos de Spodoptera frugiperda (J. E. Smith) (Lepidoptera, Noctuidae). Revista Brasileira de Entomologia 48: 119-126.

Bigler, F.; A. Meyer \& S. Bosshart. 1987. Quality assessment in Trichogramma maidis Pintureau et Voegelé reared from eggs of the factitious hosts Ephestia kuehniella Zell. and Sitotroga cerealella (Olivier). Journal of Applied Entomology 104: 340-353.

Bleicher, E. \& J. R. P. Parra. 1989. Espécies de Trichogramma parasitóides de Alabama argillacea. I. Biologia de três populações. Pesquisa Agropecuária Brasileira 24: 929-940.

Bleicher, E. \& J. R. P. Parra. 1990a. Espécies de Trichogramma parasitóides de Alabama argillacea. II. Tabela de vida de fertilidade e parasitismo de três populações. Pesquisa Agropecuária Brasileira 25: 207-214.

Bleicher, E. \& J. R. P. Parra. 1990b. Espécies de Trichogramma parasitóides de Alabama argillacea. III. Determinação das exigências térmicas de três populações. Pesquisa Agropecuária Brasileira 25: 215-219.

Butler-Junior, G. D. \& J. D. Lopez. 1980. Trichogramma pretiosum: development in two hosts in relation to constant and fluctuating temperatures. Annals of the Entomological Society America 73: 671-673.

Calvin, D. D.; M. C. Knapp; S. M. Welch; F. L. Poston \& R. J. Elzinga. 1984. Impact of environmental factors on Trichogramma pretiosum reared on southwestern corn borer eggs. Environmental Entomology 13: 774-780.

Cobert, S. A. 1985. Insect chemosensory responses: a chemical legacy hypothesis. Ecological Entomology 10: 143-153.

Cônsoli, L. F. \& J. R. P. Parra. 1996. Biology of Trichogramma galloi and T. pretiosum (Hymenoptera: Trichogrammatidae) reared in vitro and in vivo. Annals of the Entomological Society of America 89: 828-834.

Fundecitrus. 2000. Tecnologia contra o bicho-furão. Revista do Fundecitrus 96: 8-10.

Garcia, M. S. \& J. R. P. Parra. 1999. Comparação de dietas artificiais, com fontes protéicas variáveis, para criação de Ecdytolopha aurantiana (Lima, 1927) (Lepidoptera: Tortricidae). Anais da Sociedade Entomológica do Brasil 28: 219-232.

Haddad, M. L.; J. R. P. Parra \& R. C. B. Moraes. 1999. Métodos para estimar os limites térmicos inferior e superior de desenvolvimento de insetos. FEALQ, Piracicaba, 29p.

Hassan, S. A. \& M. F. Guo. 1991. Selection of effective strains of egg parasites of the genus Trichogramma (Hym., Trichogrammatidae) to control the european corn borer Ostrinia nubilalis Hübner. (Lep., Pyralidae). Journal of Applied Entomology 111: 335-341. 
Kaiser, L.; M. H. Pham-Delegue \& C. Masson. 1989. Behavioural study of plasticity in host preferences or Trichogramma maidis (Hym.: Trichogrammatidae). Physiological Entomology 14: 5360 .

Nurindah; B. W. Cribb \& G. Gordh. 1999. Experience acquisition by Trichogramma australicum Girault (Hymenoptera: Trichogrammatidae). Australian Journal of Entomology 38: $115-119$

Parra, J. R. P. 1997. Técnicas de criação de Anagasta kuehniella, hospedeiro alternativo para produção de Trichogramma, p. 121150. In: J. R. P. Parra \& R. A. Zucchi (eds.). Trichogramma e o controle biológico aplicado. Piracicaba, FEALQ, iv+324 p.

Parra, J. R. P. \& R. A. Zucchi. 1986. O uso de Trichogramma no controle de pragas, p.54-75. In: Nakano, O.; S. Silveira-Neto; J. R. P. Parra \& R. A. Zucchi (eds.). Atualização sobre métodos de controle de pragas. Piracicaba, FEALQ, 129p.

Parra, J. R. P.; P. S. M. Botelho; B. S. Corrêa-Ferreira \& J. M. S. Bento. 2002. Controle biológico uma visão inter e multidisciplinar, p.125142. In: J. R. P. Parra; P. S. M. Botelho; B. S. Corrêa-Ferreira \& J. M. S. Bento (eds.). Controle biológico no Brasil: parasitóides e predadores. São Paulo, Manole, viii +635 p.

Parra, J. R. P.; R. A. Zucchi. 2004. Trichogramma in Brazil: feasibility of use after twenty years of research. Neotropical Entomology 33: $271-281$

Pratissoli, D. \& J. R. P. Parra. 2000. Desenvolvimento e exigências térmicas de Trichogramma pretiosum Riley, criados em duas traças do tomateiro. Pesquisa Agropecuária Brasileira 35: 12811288 .
Russo, J. \& J. Voegelé. 1982. Influence de la température sur quatre espèces de trichogrammes (Hym. Trichogrammatidae) parasites de la pyrale du maïs, Ostrinia nubilalis Hübner (Lep. Pyralidae). II. - Reproduction et survie. Agronomie 2: 517-524.

Sá, L. A. N. \& J. R. P. Parra. 1994. Biology and parasitism of Trichogramma pretiosum Riley (Hym., Trichogrammatidae) on Ephestia kuehniella (Zeller) (Lep., Pyralidae) and Heliothis zea (Boddie) (Lep., Noctuidae) eggs. Journal of Applied Entomology 118: $38-43$.

Sas Institute. 1999. SAS System - SAS/STAT software: version 8.0, Cary.

Vinson, S. B. 1997. Comportamento de seleção hospedeira de parasitóides de ovos, com ênfase na família Trichogrammatidae, p.67-119. In: J. R. P. Parra \& R. A. Zucchi (eds.). Trichogramma e o controle biológico aplicado. Piracicaba, FEALQ, iii $+324 \mathrm{p}$.

Vinson, S. B. 1998. The general host selection behavior of parasitoid Hymenoptera and a comparison of initial strategies utilized by larvaphagous and oophagous species. Biological Control 11: 7996.

Wührer, B. G. \& S. A. Hassan. 1993. Selection of effective species/ strains of Trichogramma (Hym., Trichogrammatidae) to control the diamondback moth Plutella xylostella L. (Lep., Plutellidae). Journal of Applied Entomology 116: 80-89.

Yu, D. S. K.; E. A. C. Hagley \& J. E. Laing. 1984. Biology of Trichogramma minutum Riley collected from apples in southern Ontario. Environmental Entomology 13: 1325-1329. 